\title{
HOLOMORPHIC EXTENSIONS OF ORTHOGONAL PROJECTIONS INTO HOLOMORPHIC FUNCTIONS
}

\author{
J. J. KOHN ${ }^{1}$
}

ABSTRACT. A condition is given which insures that the orthogonal projection of a function into the holomorphic functions is holomorphically extendible across a given boundary point.

Recently there has been some interest in the boundary behavior of the orthogonal projection into the holomorphic functions (see [1], [2] and [3]). In [4] the writer has studied this question in terms of $C^{\infty}$ smoothness and Sobolev spaces. In the present note we point out that the analyticity at the boundary of the projection is connected with the solvability of an equation of the type studied by $\mathrm{H}$. Lewy (see [5]). The deep problem in the study of analyticity is to establish Condition I (which is formulated below). This condition is satisfied in complex dimension one (see [6]). In higher dimensions it is easily verified for those (few) domains for which the kernel function is known explicitly.

Let $\Omega$ be an open subset of $\mathbf{C}^{n}$ (or of a complex hermitian manifold) with $\bar{\Omega}$, the closure of $\Omega$, compact. Let $L_{2}(\Omega)$ and $\mathcal{H}(\Omega)$ stand for the spaces of complex-valued square-integrable functions on $\Omega$ and holomorphic square-integrable functions on $\Omega$, respectively. We denote by $H: L_{2}(\Omega) \rightarrow$ $\mathcal{H}(\Omega)$ the orthogonal projection of $L_{2}(\Omega)$ onto the subspace $\mathcal{H}(\Omega)$. Given $f \in L_{2}(\Omega)$ and $z^{0} \in b \Omega$ (where $b \Omega$ denotes the boundary of $\Omega$ ) it is natural to ask whether $H f$ has a holomorphic extension across $z^{0}$; more precisely: does there exist a neighborhood $V$ of $z^{0}$ and a holomorphic function $h$ on $\Omega \cup V$, such that $h=H f$ in $V$. Now $H f$ can be expressed by:

$$
(H f)(z)=\int_{\Omega} K_{\boldsymbol{\Omega}}(z, \bar{w}) f(w) d V_{w},
$$

where $K_{\Omega}(z, \bar{w})$ is the Bergman kernel function of the domain $\Omega$.

Remark. If for every neighborhood $U_{0}$ of $z^{0}$ there exists a neighborhood $U_{1}$ of $z^{0}$ such that for every $f \in L_{2}(\Omega)$ which vanishes on $\Omega \cap U_{0}$, $H f$ extends to a holomorphic function on $\Omega \cup U_{1}$ then $K_{\Omega}(z, \bar{w})$ satisfies

Condition I. If $U_{0}$ is a neighborhood of $z^{0}$ then there exists a neighborhood $U_{1}$ of $z^{0}$ such that for each fixed $w^{0} \in \Omega$ and $w \notin U_{0}$ the function $K_{\Omega}\left(z, \bar{w}^{0}\right)$ extends to a holomorphic function on $\Omega \cup U_{1}$.

Received by the editors June 27, 1974.

AMS (MOS) subject classifications (1970). Primary 32A10, 32H10.

1 During the preparation of this paper the author has been partially supported by an NSF research project at Princeton University. 
To prove the Remark it suffices to find a $g \in C_{0}^{\infty}(\Omega)$ whose support lies outside of $U_{0}$ such that for $z \in U_{1}$ we have

$$
(H g)(z)=K_{\mathbf{\Omega}}\left(z, \bar{w}^{0}\right) \text {. }
$$

This can be done (as in [2] and [3]) by choosing $\epsilon$ suitably small, and setting:

$$
g(z)=\epsilon^{-2 n} \chi\left(\left(z-w^{0}\right) / \epsilon\right)
$$

where $\chi$ is a nonnegative $C^{\infty}$ function with support in the unit ball, such that $\int \chi(\zeta) d V_{\zeta}=1$ and $\chi$ depends only on $|\zeta|$. It then suffices to prove (2) for $z \in \Omega$. This follows immediately on setting $z_{j}=r_{j} e^{i \theta_{j}}-w_{j}^{0}$ and integrating with respect to the coordinates $r_{1}, \ldots, r_{n}, \theta_{1}, \ldots, \theta_{n}$.

Assume that in a neighborhood $U$ of $z^{0} \in b \Omega$ the boundary is smooth in the sense that there exists a function $r \in C^{\infty}(U)$ such that $d r \neq 0$ and $r=$ 0 on $U \cap b \Omega$. We wish to impose a condition on the restriction of $f$ to $U$ $\cap \Omega$ which will insure that $H f$ can be extended holomorphically across $z^{0}$. This condition is formulated below.

Condition II. Let $A$ be an operator on $U$ defined by

$$
A=\sum_{1}^{n} a_{j} \frac{\partial}{\partial \bar{z}_{j}}
$$

where $a_{j} \in C^{\infty}(U)$ and

$$
A(r)=0 \text { on } U \cap b \Omega
$$

We say condition $f \in L_{2}(\Omega)$ satisfies Condition II on $U \cap \Omega$ if there exists $u \in L_{2}(U \cap \Omega)$ such that for some operator $A$ of the type defined by (4) and (5) we have

$$
A^{*}(u)=f,
$$

where $A^{*}$ denotes the formal adjoint of $f$ and is given by

$$
A^{*}(u)=-\sum_{1}^{n} \bar{a}_{j} \frac{\partial u}{\partial z_{j}}-\left(\sum_{1}^{n} \frac{\partial \bar{a}_{j}}{\partial z_{j}}\right) u .
$$

Theorem. Suppose Condition I holds for $K_{\Omega}$ and $f \in L_{2}(\Omega)$ satisfies Condition II in $U \cap \Omega$, where $U$ is a neighborhood of $z^{0}$. Then there exists a neighborhood $U_{1}$ of $z^{0}$ such that $H f$ has a holomorphic extension to $\Omega$ $\cup U_{1}$.

Before proceeding with the proof of the above Theorem, we observe that the following is one of its consequences.

Corollary. Suppose that the function $r$ which defines the boundary in a neighborhood $U$ of $z^{0}$ (i.e. $r=0$ on $U \cap b \Omega$ and $d r \neq 0$ ) is real-analytic. 
Then Condition I is equivalent to the following. For every neighborhood $U_{0}$ of $z^{0}$ there exists a neighborhood $U_{1}$ of $z^{0}$ such that whenever $f \epsilon$ $L_{2}(\Omega)$ has an extension $\tilde{f}$ to $\Omega \cup U_{0}$ with $\tilde{f}$ analytic ${ }^{2}$ on $U_{0}$ then $H f$ can be holomorphically extended to $\Omega \cup U_{1}$.

Proof of Corollary. The necessity of Condition I follows from the above Remark. To show that Condition $\mathrm{I}$ is sufficient we define $A$ on $U$ by:

$$
A=\frac{\partial r}{\partial \bar{z}_{2}} \frac{\partial}{\partial \bar{z}_{1}}-\frac{\partial r}{\partial \bar{z}_{1}} \frac{\partial}{\partial \bar{z}_{2}},
$$

then $A^{*}$ has analytic ${ }^{2}$ coefficients and hence the equation (6) has a solution by the Cauchy-Kowalevsky theorem.

Observe that $A$ given by (8) operates on functions which are defined on the surfaces $r$ = constant. Thus the assumptions on $f$ in the Corollary can be considerably relaxed. It would suffice to assume that the restriction of $f$ to each one of the surfaces is analytic ${ }^{2}$ in a sufficiently uniform way so that application of the Cauchy-Kowalevsky theorem on each surface will yield a solution of (6).

Proof of the Theorem. Let $\zeta \in C_{0}^{\infty}(U)$ such that $\zeta=1$ on a neighborhood $U_{0}$ of $z^{0}$. Define $v$ on $\Omega$ by

$$
v= \begin{cases}A^{*}(\zeta u) & \text { on } U \cap \Omega, \\ 0 & \text { on } \Omega-U \cap \Omega .\end{cases}
$$

Then $v \in L_{2}(\Omega)$, since on $U$, , $\Omega$ we have

$$
v=\zeta A^{*} u-\left(\sum_{1}^{n} \bar{a}_{j} \frac{\partial \zeta}{\partial z_{j}}\right) u
$$

Now if $h \in L_{2}(\Omega)$ such that $A h \in L_{2}\left(\Omega r_{1} U\right)$ then, by integration by parts we obtain

$$
(v, h)=\int_{\Omega \cap U} \zeta u(\overline{A h}) d V
$$

there is no boundary term because of (5). Thus we see that $v$ is orthogonal to $\mathcal{H}(\Omega)$, since $A h=0$ whenever $h$ is holomorphic.

Setting

$$
g=f-v
$$

we have $H(g)=H(f)$ and by (6) and (9) we have $g=0$ in $U_{0}$. Hence we have

$$
(H f)(z)=\int_{\Omega-\Omega \cap U_{0}} K_{\Omega}(z, \vec{w}) g(w) d V_{w}
$$
functions.

2 By analytic we mean that the real and imaginary parts are real-analytic 
and therefore, by Condition I, $H f$ has holomorphic extension to $\Omega \cup U_{1}$.

Finally we observe that the solvability of (6) is not necessary for the required extension. For if the Levi-form at $z^{0}$ has a negative eigenvalue, then every holomorphic function (and in particular $H f$ ) can be holomorphically extended across $z^{0}$.

\section{REFERENCES}

1. C. Fefferman, The Bergman kernel and biholomorphic mappings of pseudoconvex domains, Communications in P. D. E. (to appear).

2. L. Hörmander, The boundary behaviour of the Bergman kernel (preprint).

3. N. Kerzman, The Bergman kernel function: differentiability at the boundary, Math. Ann. 195 (1972), 149-158.

4. J. J. Kohn, Harmonic integrals on strongly pseudoconvex manifolds. I, II, Ann. of Math. (2) 78 (1963), 112-148; ibid. 79 (1964), 450-472. MR 27 \#2999; 34 $\# 8010$.

5. H. Lewy, $O_{n}$ the local character of the solutions of an atypical linear differential equation in three variables and a related theorem for regular functions of two complex variables, Ann. of Math. (2) 64 (1956), 514-522. MR 18, 473.

6. S. Bergman, The kernel function and conformal mapping, Math. Surveys, no. 5, Amer. Math. Soc, Providence, R. I., 1950. MR 12, 402.

7. E. Stein, Boundary behavior of holomorphic functions of several complex variables, Math. Notes, Princeton Univ. Press, Princeton, N. J., 1972.

DEP ARTMENT OF MATHEMATICS, PRINCETON UNIVERSITY, PRINCETON, NEW JER. SEY 08540 